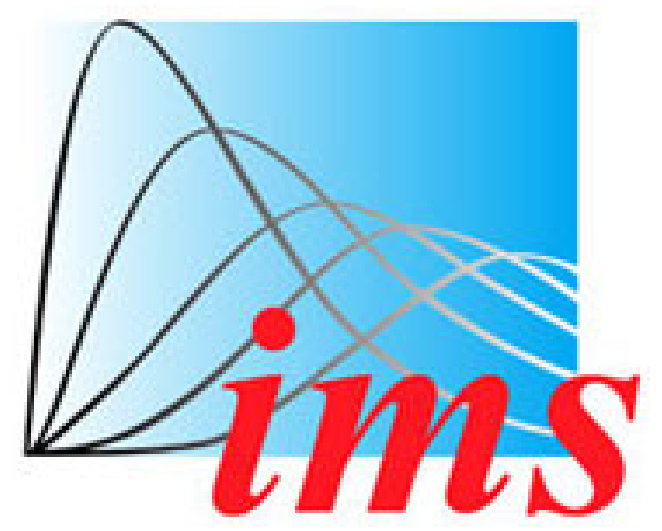

Merging of Opinions with Increasing Information

Author(s): David Blackwell and Lester Dubins

Source: The Annals of Mathematical Statistics, Vol. 33, No. 3 (Sep., 1962), pp. 882-886

Published by: Institute of Mathematical Statistics

Stable URL: http://www.jstor.org/stable/2237864

Accessed: 08/12/2010 11:08

Your use of the JSTOR archive indicates your acceptance of JSTOR's Terms and Conditions of Use, available at http://www.jstor.org/page/info/about/policies/terms.jsp. JSTOR's Terms and Conditions of Use provides, in part, that unless you have obtained prior permission, you may not download an entire issue of a journal or multiple copies of articles, and you may use content in the JSTOR archive only for your personal, non-commercial use.

Please contact the publisher regarding any further use of this work. Publisher contact information may be obtained at http://www.jstor.org/action/showPublisher?publisherCode=ims.

Each copy of any part of a JSTOR transmission must contain the same copyright notice that appears on the screen or printed page of such transmission.

JSTOR is a not-for-profit service that helps scholars, researchers, and students discover, use, and build upon a wide range of content in a trusted digital archive. We use information technology and tools to increase productivity and facilitate new forms of scholarship. For more information about JSTOR, please contact support@jstor.org. 


\title{
MERGING OF OPINIONS WITH INCREASING INFORMATION ${ }^{1}$
}

\author{
By David Blackwell and Lester Dubins \\ University of California, Berkeley
}

1. History. One of us [1] has shown that if $Z n, n=1,2, \ldots$ is a stochasticprocess with $D$ states, $0,1, \cdots, D-1$ such that $X=\sum_{n=1}^{\infty} Z_{n} / D^{n}$ has an absolutely continuous distribution with respect to Lebesgue measure, then the conditional distribution of $R_{k}=\sum_{n=1}^{\infty} Z_{k+n} / D^{n}$ given $Z_{1}, \cdots, Z_{k}$ converges with probability one as $k \rightarrow \infty$ to the uniform distribution on the unit interval, in the sense that for each $\lambda, 0<\lambda \leqq 1, P\left(R_{k}<\lambda \mid Z_{1}, \cdots, Z_{k}\right) \rightarrow \lambda$ with probability 1 as $k \rightarrow \infty$. It follows that the unconditional distribution of $R_{k}$ converges to the uniform distribution as $k \rightarrow \infty$. If $\left\{Z_{n}\right\}$ is stationary, the distribution of $R_{k}$ is independent of $k$, and hence uniform, a result obtained earlier by Harris [3]. Earlier work relevant to convergence of opinion can be found in [4, Chap. 3, Sect. 6].

Here we generalize these results and also show that the conditional distribution of $R_{k}$ given $Z_{1}, \cdots, Z_{k}$ converges in a much stronger sense. All probabilities. in this paper are countably additive.

2. Statement of the theorem. Let $\beta_{i}$ be a $\sigma$-field of subsets of a set $X_{i}$, $i=1,2, \cdots$; and let $(X, \beta)=\left(X_{1} \times X_{2} \times \cdots, B_{1} \times B_{2} \times \cdots\right)$. Suppose $(X, \beta, P)$ is a probability space and let $P_{n}$ be the marginal distribution of $\left(X_{1} \times \cdots \times X_{n}, \bigotimes_{1} \times \cdots \times \bigotimes_{n}\right)$; that is, $P_{n}(A)=P\left(A \times X_{n+1} \times \cdots\right)$ for all $A \in B_{1} \times \cdots \times \bigotimes_{n}$. The probability $P$ is predictive if for every $n \geqq 1$, there exists a conditional distribution $P^{n}$ for the future $X_{n+1} \times \cdots$ given the past $X_{1}, \cdots, X_{n}$; that is, if there exists a function $P^{n}\left(x_{1}, \cdots, x_{n}\right)(C)$ where $\left(x_{1}, \cdots, x_{n}\right)$ ranges over $X_{1} \times \cdots \times X_{n}$ and $C$ ranges over $B_{n+1} \times \cdots$ with the usual three properties: $P^{n}\left(x_{1}, \cdots, x_{n}\right)(C)$ is $\beta_{1} \times \cdots \times \beta_{n}$-measurable for fixed $C$; a probability distribution on $\left(X_{n+1} \times \cdots ; \Theta_{n+1} \times \cdots\right)$ for fixed $\left(x_{1}, \cdots, x_{n}\right)$; and for bounded $ß$-measurable $\phi$

$$
\begin{aligned}
\int \phi d P=\int\left[\left(\phi\left(x_{1}, \cdots, x_{n}, x_{n+1}, \cdots\right) d P^{n}\left(x_{n+1}, \cdots \mid x_{1}, \cdots, x_{n}\right)\right]\right. \\
\cdot d P_{n}\left(x_{1}, \cdots, x_{n}\right)
\end{aligned}
$$

holds.

The assumption that $P$ is predictive is mild and applies to all natural probabilities known to us. It is easy to verify that any probability which is absolutely continuous with respect to a predictive probability is also predictive.

Received December 12, 1961.

1 This paper was prepared with the partial support of the Office of Naval Research (Nonr-222-43) for Mr. Blackwell; and with the partial support of the National Science Foundation, Grant G-14648 for Mr. Dubins. This paper in whole or in part may be reproduced for any purpose of the United States Government. 
For any two probabilities $\mu_{1}$ and $\mu_{2}$ on the same $\sigma$-field $\mathcal{F}$, the well known distance $\rho\left(\mu_{1}, \mu_{2}\right)$ between $\mu_{1}$ and $\mu_{2}$ is the least upper bound over $D \varepsilon \mathcal{F}$ of $\left|\mu_{1}(D)-\mu_{2}(D)\right|$. Of course $\mu_{i}$ is absolutely continuous with respect to $\left(\mu_{1}+\right.$ $\left.\mu_{2}\right) / 2=m$ and has a density $\phi_{i}$, so that $\rho\left(\mu_{1}, \mu_{2}\right)=\int_{A}\left(\phi_{1}-\phi_{2}\right) d m=$ $(1 / 2) \int\left|\phi_{1}-\phi_{2}\right| d m$ where $A$ is the set where $\phi_{1}-\phi_{2}>0$.

MAIN Theorem. Suppose that $P$ is a predictive probability on $(X, \Theta)$ and that $Q$ is absolutely continuous with respect to $P$. Then for each conditional distribution $P^{n}$ of the future given the past with respect to $P$, there exists a conditional distribution $Q^{n}$ of the future given the past with respect to $Q$ such that, with the exception of a set of histories $\left(x_{1}, \cdots, x_{n}, x_{n+1}, \cdots\right)$ of Q-probability 0 , the distance between $P^{n}\left(x_{1}, \cdots, x_{n}\right)$ and $Q^{n}\left(x_{1}, \cdots x_{n}\right)$ converges to 0 as $n$ converges to $\infty$.

3. Martingale preliminaries. The proof of the theorem requires a slightly generalized martingale convergence theorem. Say that a sequence $\left\{y_{n}\right\}$ of random variables is dominated in the sense of Lebesgue if $\sup _{n}\left|y_{n}\right|$ has a finite expectation.

Theorem 2. Suppose that $\left\{y_{n}\right\}, n=1,2, \cdots$, a sequence of random variables dominated in the sense of Lebesgue, converges almost everywhere to a random variable $y$. Then for every monotone increasing or monotone decreasing sequence of $\sigma$-fields $\mathcal{u}_{j}, j=1,2, \cdots$ converging to a $\sigma$-field $\mathfrak{u}$,

$$
\lim _{\substack{j \rightarrow \infty \\ n \rightarrow \infty}} E\left[y_{n} \mid \mathcal{u}_{j}\right]=E[y \mid \mathcal{u}]
$$

almost everywhere and in $L_{1}$.

In this note we are primarily interested in the weaker conclusion that $\lim _{n \rightarrow \infty} E\left[y_{n} \mid \mathcal{u}_{n}\right]=E[y \mid \mathcal{u}$. The two important special cases in which either $y_{n}$ or $\mathfrak{u}_{n}$ is independent of $n$ are in [2].

Proof of Theorem 2 . Let $g_{k}=\sup y_{n}$ for $n \geqq k$. Equalities and inequalities below are asserted to hold with probability 1 . Fix $k$ for a moment and let $n \geqq k$. Then $y_{n} \leqq g_{k}$ and

$$
E\left[y_{n} \mid \mathfrak{u}_{i}\right] \leqq E\left[g_{k} \mid \mathfrak{u}_{i}\right]
$$

Letting

$$
\begin{aligned}
& z=\lim _{j} \sup _{\substack{i \geqq j \\
n \geqq j}} E\left[y_{n} \mid \mathfrak{u}_{i}\right], \\
& x=\lim _{j} \inf _{\substack{i \geqq j \\
n \geqq j}} E\left[y_{n} \mid \mathfrak{u}_{i}\right],
\end{aligned}
$$

you conclude from (3) and a usual form of martingale convergence theorem [For example, see 2, Theorem 4.3, Chap. VII] that

$$
z \leqq \lim _{j} \sup _{i \geqq j} E\left[g_{k} \mid \mathfrak{u}_{i}\right]=\lim _{i} E\left[g_{k} \mid \mathcal{u}_{i}\right]=E\left[g_{k} \mid \mathcal{u}\right] .
$$

Therefore $z \leqq \lim E\left[g_{k} \mid \mathcal{u}\right]=E[y \mid \mathcal{u}]$ by Lebesgue's theorem suitably generalized so as to apply to conditional expectations. [See, for example, 2, $\mathrm{CE}_{5}$ Section 8, Chap. 1]. Similarly, $x \geqq E[y \mid \mathcal{u}$, and the proof of almost everywhere convergence is complete. The proof of $L_{1}$ convergence is routine and omitted. 
Corollary 1. Suppose that with probability 1, only a finite number of the events $E_{1}, E_{2}, \cdots$ occur. Then for any monotone sequence of $\sigma$-fields $\mathfrak{u}_{1}, \mathfrak{u}_{2}, \cdots$

$$
P\left[\bigcup_{k \geqq n} E_{k} \mid \mathfrak{u}_{j}\right] \text { and } P\left[E_{n} \mid \mathfrak{u}_{j}\right] \rightarrow 0 .
$$

almost surely as $n$ and $j \rightarrow \infty$.

CoRollary 2. If $f_{n}$ is any sequence of random variables that converges almost everywhere to 0 and $\mathcal{u}_{j}$ is a monotone sequence of $\sigma$-fields, then with probability 1 , for all $\epsilon>0$,

$$
P\left[\sup _{k \geqq n}\left|f_{k}\right|>\epsilon \mid \mathcal{u}_{j}\right], \text { and } P\left[\left|f_{n}\right|>\epsilon \mid \mathfrak{u}_{j}\right]
$$

converge to 0 as $n$ and $j$ converge to $\infty$.

CoRollaRY 3. Let $q \geqq 0$ be a density function for which $Q(B)=\int_{B} q d P$ for all $B \varepsilon B ;$ let

$$
q_{n}\left(x_{1}, \cdots, x_{n}\right)=\int q\left(x_{1}, \cdots, x_{n}, x_{n+1}, \cdots\right) d P^{n}\left(x_{n+1}, \cdots \mid x_{1}, \cdots, x_{n}\right) ;
$$

and let

$$
\begin{array}{r}
d_{n}\left(x_{1}, \cdots, x_{n}, x_{n+1}, \cdots\right)=q\left(x_{1}, \cdots, x_{n}, x_{n+1}, \cdots\right) / \\
q_{n}\left(x_{1}, \cdots, x_{n}\right) \text { or } 1,
\end{array}
$$

according as $q_{n}\left(x_{1}, \cdots, x_{n}\right) \neq 0$ or not. Then, with P-probability 1 , for all $\epsilon>0$,

$$
P\left[d_{n}-1>\epsilon \mid x_{1}, \cdots, x_{n}\right] \rightarrow 0 \text { as } n \rightarrow \infty,
$$

and with Q-probability 1, for all $\epsilon>0$,

$$
Q\left[\left|d_{n}-1\right|>\epsilon \mid x_{1}, \cdots, x_{n}\right] \rightarrow 0 \text { as } n \rightarrow \infty .
$$

Proof of Corollary 3. With respect to $P$ measure,

$$
E\left[q \mid x_{1}, \cdots, x_{n}\right]=q_{n}\left(x_{1}, \cdots, x_{n}\right),
$$

so that according to Doob's martingale convergence theorem, $q_{n}\left(x_{1}, \cdots, x_{n}\right)$ converges to $q\left(x_{1}, \cdots, x_{n}, x_{n+1}, \cdots\right)$ almost surely with respect to $P$. Consequently, $\varlimsup d_{n} \leq 1$ a.s. $P$ and $d_{n} \rightarrow 1$ a.s. $Q$ since $q>0$ a.s. $Q$. An application of Corollary 2 completes the proof.

4. Proof of main theorem. Define

$$
\begin{aligned}
& Q^{n}\left(x_{1}, \cdots, x_{n}\right)(C) \\
& \quad=\int_{C} d_{n}\left(x_{1}, \cdots, x_{n}, x_{n+1}, \cdots\right) d P^{n}\left(x_{n+1}, \cdots \mid x_{1}, \cdots, x_{n}\right),
\end{aligned}
$$

for all $C \varepsilon B_{n+1} \times \cdots$. 
It is routine to verify that $Q^{n}$ is a conditional distribution for the future given the past. Let $u=\left(x_{1}, \cdots, x_{n}\right)$ and $v=\left(x_{n+1}, \cdots\right)$, and compute thus:

$$
\begin{aligned}
\rho\left(P^{n}\left(x_{1}, \cdots, x_{n}\right)\right. & \left., Q^{n}\left(x_{1}, \cdots, x_{n}\right)\right) \\
= & \rho\left(P^{n}(u), Q^{n}(u)\right) \\
& =\int\left(d_{n}(u, v)-1\right) d P^{n}(v \mid u) \text { over } v: d_{n}(u, v)-1>0 \\
& \leqq \epsilon+\int d_{n}(u, v) d P^{n}(v \mid u) \text { over } v: d_{n}(u, v)-1>\epsilon \\
& =\epsilon+Q^{n}(u)\left(v: d_{n}(u, v)-1>\epsilon\right) \\
& =\epsilon+Q\left[d_{n}-1>\epsilon \mid x_{1}, \cdots, x_{n}\right] \\
& =\epsilon+\epsilon
\end{aligned}
$$

for all but a finite number of $n$ with $Q$-probability 1, according to (11). This completes the proof.

5. Interpretation. Usually, there is essentially only one conditional distribution $Q^{n}$ of the future given the past. Therefore, our theorem may be interpreted to imply that if the opinions of two individuals, as summarized by $P$ and $Q$, agree only in that $P(D)>0 \leftrightarrow Q(D)>0$, then they are certain that after a sufficiently large finite number of observations $x_{1}, \cdots, x_{n}$, their opinions will become and remain close to each other, where close means that for every event $E$ the probability that one man assigns to $E$ differs by at most $\epsilon$ from the probability that the other man assigns to it, where $\epsilon$ does not depend on $E$. Leonard J. Savage observed that our theorem applies to the particularly interesting case in which $P$ and $Q$ are symmetric (or exchangeable). That is, if the measures $P$ and $Q$ on the sequences $x_{i}$ are those that arise when the $x_{i}$ are, for a fixed parameter value, independent and identically distributed observations, with prior distributions $p$ and $q$ on the parameter, then the relations of absolute continuity between $P$ and $Q$ are precisely those between $p$ and $q$.

6. Caution. Though the conditional distributions of the future $P^{n}$ and $Q^{n}$ merge as $n$ becomes large, this need not happen to the unconditional distributions of the future. That is, let $P(n)(D)=P\left(X_{1} \times \cdots \times X_{n} \times D\right)$ for all $D \varepsilon \leftrightarrow_{n+1} \times \cdots$, and let $Q(n)$ be similarly defined. The following is a simple example of two probabilities $P$ and $Q$ absolutely continuous with respect to each other for which $P(n)$ and $Q(n)$ do not merge with increasing $n$. Let $R$ be the probability on infinite sequences $x_{1}, x_{2}, \cdots$ of 0 's and 1's determined by independent tosses of a coin which has probability $r$ of success, and let $S$ be the probability determined if the coin has probability $s$ for success, with $0 \leqq r \leqq 1$, $0 \leqq s \leqq 1$, and $r \neq s$. Now let $0<p<q<1$ and let $P$ and $Q$ be mixtures of $R$ and $S: P=p R+(1-p) S, Q=q R+(1-q) S$. Since $P(n)=P$ and $Q(n)=Q$ for all $n$, there is no tendency for $P(n)$ and $Q(n)$ to merge. 
7. An application. By viewing the unit interval as a product of two point spaces, the interested reader will see that the main theorem yields information about the local behavior of positive integrable functions $q(x)$ defined for $0 \leqq x \leqq 1$.

\section{REFERENCES}

[1] Blackwell, David (1957). On discrete variables whose sum is absolutely continuous. Ann. Math. Statist. 28 520-521.

[2] Dooв, J. L. (1953). Stochastic Processes. Wiley, New York.

[3] Harris, T. E. (1955). On chains of infinite order. Pacific J. Math. 5 707-724.

[4] Savage, Leonard J. (1954). The Foundations of Statistics. Wiley, New York. 